Deni Vlašić

Nastia Degiuli

Andrea Farkas

Ivana Martić

http://dx.doi.org/10.21278/brod69308

\title{
THE PRELIMINARY DESIGN OF A SCREW PROPELLER BY MEANS OF COMPUTATIONAL FLUID DYNAMICS
}

UDC 629.5.016: 629.5.035

Original scientific paper

\begin{abstract}
Summary
The assessment of hydrodynamic characteristics of a screw propeller in open water test is of crucial significance in the preliminary design stage of a ship. The open water characteristics can be accurately predicted by towing tank measurements. Taking into account the significant advances in computational fluid dynamics which has enabled the numerical assessment of the open water characteristics, the time and the cost of propeller design are significantly reduced. Open water characteristics can be assessed numerically using potential and viscous flow theory. The former one allows faster and simpler preliminary determination of open water characteristics. Within this paper, numerical simulations are performed for Gawn series propellers utilizing open source code OpenProp based on the moderately loaded lifting line theory and commercial software package STAR-CCM+ based on viscous flow theory. The latter one is more time consuming regarding the time required for the preparation of simulation as well as computational time. The obtained numerical results are compared with regression polynomials based on the experimental data. The validation of the results has pointed out that OpenProp can be used as practical and efficient tool in preliminary design of screw propellers.
\end{abstract}

Key words: $\quad$ screw propeller; open water test; potential flow theory; viscous flow theory

\section{Introduction}

The most important phase in the design of a screw propeller is the preliminary design phase during which the geometry of the screw propeller is chosen, in order to ensure the required thrust at a certain ship speed. Nowadays, the hydrodynamic characteristics of a screw propeller are most commonly determined utilizing experimental methods, i.e. open water test (OWT) is performed [1]. In the past different approaches based on the potential flow theory have been developed for modelling of the flow around a screw propeller. Numerical methods for the determination of the hydrodynamic characteristics of a screw propeller are according to the order of complexity [2]: momentum theory, lifting line method, lifting-surface method, Boundary Element Method (BEM) or panel method and Reynolds Averaged Navier-Stokes 
(RANS) equations. Numerical simulations based on the potential flow theory provide a rapid estimation of the hydrodynamic characteristics of a screw propeller in OWT and therefore are of great benefit to ship designers. The lifting line method is the fastest and easiest to apply, as it does not require a complex preparation of the three-dimensional representation of a screw propeller. Because of its computational efficiency, it has been a key factor for a number of years in the preliminary design of a screw propeller [3]. On the other hand, methods for the determination of the hydrodynamic characteristics of a screw propeller based on the viscous flow theory are used as well. With the development of computers in last years these methods have taken an increasingly important role in screw hydrodynamics.

The possibilities of improving the geometry of a screw propeller at the preliminary design stage using methods based on the potential flow theory are presented in [4]. Validation of the results was performed on DTMB 4119 and DTMB 4381 screw propellers for which the experimental data were available. The proposed method showed that the geometry of the DTMB 4119 screw propeller was optimal. Furthermore, geometric improvements had been achieved by the rearrangement of the pitch along the blade of a screw propeller by applying the same method for the DTMB 4381 screw propeller. In this way, a more favorable distribution of circulation along the blades of a screw propeller was achieved. Gaggero et al. [5] have performed the geometry optimization of the Contracted and Tip Loaded (CLT) propeller using BEM. The reliability of the design method and achieved improvements in the screw propeller geometry had been validated by extensive RANS calculations. The authors also performed unsteady calculations using unsteady BEM to obtain amplitudes of induced pressure impulses. The obtained results were compared with the available measurements and with results for the geometry of original CLT propeller and the authors confirmed the improvements for the new geometry. Bertetta et al. [6] have investigated the influence of the cavitation on the noise for a Controlable Pitch Propeller (CPP) outside the operating point using BEM. The multiobjective optimization algorithm coupled with BEM led to the optimum geometry of CPP for different pitches with the aim of reducing cavitation and noise. Outside the operating point of the screw propeller, the new geometry produces less noise and less pressure impulses than the original geometry. Lee et al. [7] have proposed an optimization code coupled with the lifting-surface method for the design of a screw propeller. The method uses a vortex theory to calculate the induced speeds at certain radii of a screw propeller. The validation of the results was carried out on DTNSRDC 4119 propeller with a homogeneous velocity field as well as on a series of DTNSRDC propellers that had more complex geometry. A study of the hydrodynamic characteristics of a screw propeller, operating near the free surface, for different advance coefficients and various submergence of propeller models was performed in [8]. The impact of the scale effects was evaluated by testing two models in different scale. The results of the numerical simulation included the free surface pattern, the velocity field around the screw propeller, and the hydrodynamic characteristics of the screw propeller. Islam et al. [9] have studied the influence of domain size and discretization parameters on the time required to run RANS simulation of OWT in the commercial software package STAR-CCM+ as well as their impact on the accuracy of the results. Based on the results for 32 different domains for the same screw propeller, the authors found the optimum domain dimension and domain discretization parameters utilizing design of experiments. The obtained results were validated by comparison with the experimental data. Califano and Steen [10] have performed RANS simulations using the commercial software package Fluent to investigate the effects of ventilation on the hydrodynamic 
characteristics of a screw propeller while operating in the heavy sea. The authors concluded that predicted dynamic loads utilizing numerical simulations showed satisfactory agreement with the experimentally obtained ones, but only at the upright position where the blade is piercing the free surface. For the other angular positions the thrust is overestimated. This was attributed to the inability of RANS solver to resolve the tip vortex. Furthermore, it was shown that the tip vortex had a very important role in the ventilation of conventional propellers, which was not a case for surface-piercing propellers. Subhas et al. [11] have carried out a numerical simulation of OWT using RANS solver in Fluent for the prediction of the pressure field and the velocity around the screw propeller, as well as the cavitation occurrence. The comparison of the obtained results with experimental ones showed that Computational Fluid Dynamics (CFD) can be used for prediction of the cavitation occurrence. Prakash and Nath [12] have performed a numerical simulation of OWT for a four-blade propeller of Wageningen B series in Fluent utilizing unstructured grid. The validation of the obtained results was performed by comparison with the results obtained using regression polynomials which were derived based on experimental results. Krasilnikov et al. [13] have investigated the scale effects utilizing RANS simulations with the main focus on the impact of blade skew, propeller loading and blade area ratio. Müller et al. [14] have analyzed the impact of the scale effects on screw propeller for large container ships. The authors performed the numerical simulations of OWT within commercial software package CFX for 23 screw propellers in model scale and full-scale. Based on the obtained results, they proposed a scaling method for evaluation of OWT characteristics in full-scale. Lee and Paik [15] have performed numerical simulations of partially submerged screw propeller under the bollard condition. The authors showed that thrust and torque of partially submerged propeller decrease significantly with an increase in rate of revolution. In [16], the author has investigated wake characteristics of Contra Rotating Propeller (CRP) in OWT and self-propulsion conditions. In addition, Paik studied the effect of rudder on wake characteristics in self-propulsion test and concluded that the presence of the rudder has no significant influence on wake whereas a significant influence on thrust and torque was noticed. Kinaci and Gokce [17] have studied effect of propeller on free surface elevations for benchmark Post Panamax ship, Duisburg test case. For this purpose, the authors performed numerical simulations of resistance and self-propulsion tests. The authors concluded that free surface causes the increase of pressure on the propeller and hull stern as well as the efficiency of the propeller.

In this paper OWT characteristics are studied numerically utilizing lifting line theory and RANS. Numerical simulations are performed for five Gawn series propellers with expanded area ratio equal to 1.1. Pitch to diameter ratio of propellers with three blades is varied in the range from 0.7 to 0.9 . The obtained numerical results are compared with regression polynomials based on the experimental data. Furthermore, the flow around the screw propeller in OWT is analyzed. This paper is organized as follows: Section 2 provides governing equations for lifting line and viscous flow theory, while in Section 3 numerical setup is given. Section 4 provides the obtained results, while in Section 5 conclusions drawn from this research are given. 


\section{Governing equations}

\subsection{Lifting line theory}

This subsection presents the theory of moderately loaded lifting line enhanced with vortex lattice for computing the induced velocities. Propeller blade is represented by a lifting line, with trailing vorticity aligned to the local flow velocity. The induced velocities are computed using a vortex lattice theory, with helical trailing vortex filaments shed at discrete stations along the blade. The blade itself is modelled as discrete sections, having $2 \mathrm{D}$ section properties at each radius. Loads are computed by integrating the $2 \mathrm{D}$ section loads over the span of the blade [18].

Using the lifting and viscous forces for infinitely many radial sections along the propeller blades, i.e. knowing the dependence of these two forces on the radial position along the propeller blades $(r)$ and under the assumption of identical propeller blades, the total thrust and torque can be calculated as follows [18]:

$$
\begin{aligned}
& \mathbf{T}=Z \int_{r_{h}}^{R}\left[F_{i} \cos \left(\beta_{i}\right)-F_{v} \sin \left(\beta_{i}\right)\right] \mathrm{d} r\left(\mathbf{e}_{\mathbf{a}}\right) \\
& \mathbf{Q}=Z \int_{r_{h}}^{R}\left[F_{i} \cos \left(\beta_{i}\right)+F_{v} \sin \left(\beta_{i}\right)\right] r \mathrm{~d} r\left(-\mathbf{e}_{\mathbf{a}}\right)
\end{aligned}
$$

where $Z$ is the number of blades, $F_{i}$ is the magnitude of lifting force, $F_{v}$ is the magnitude of viscous force, $\beta_{i}$ is the angle of the resultant inflow velocity $\left(\mathrm{V}^{*}\right), \mathbf{e}_{\mathrm{a}}$ is the axial direction, $r_{h}$ is the propeller hub radius and $R$ is the radius of the propeller. Figure 1 shows the forces and velocities acting on the blade section.

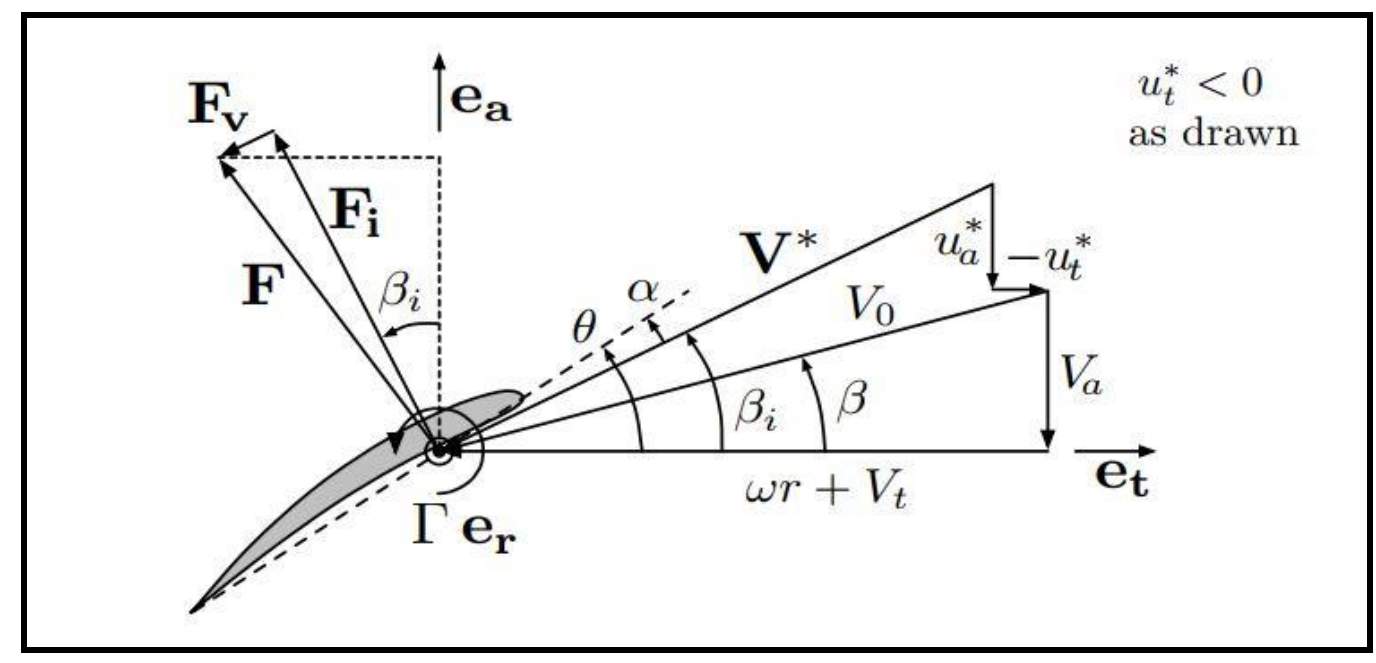

Fig. 1 Forces and velocities acting on the blade section, [18]

The magnitudes of lifting and viscous forces can be determined as follows:

$$
F_{i}=\rho V^{*} \Gamma
$$




$$
F_{v}=\frac{1}{2} \rho\left(V^{*}\right)^{2} C_{D} c
$$

where $\rho$ is the fluid density, $V^{*}$ is the total resultant inflow velocity, $\Gamma$ is the magnitude of circulation, $C_{D}$ is the section drag coefficient and $c$ is the section chord. The section drag coefficient $C_{D}$ is determined by means of charts for known hydrodynamic profile and Reynolds number calculated on the basis of the chord length.

The circulation is computed from 2D lift coefficient, which is given as follows:

$$
C_{L}=\frac{2 \Gamma}{V^{*} c}
$$

A standard propeller vortex lattice model is used to compute the axial and tangential induced velocities $\left(u_{a}^{*}, u_{t}^{*}\right)$. In the vortex lattice formulation, a propeller with $Z$ blades is modelled as a single representative radial lifting line, partitioned into $M$ panels. The induced velocities are computed at control points on the lifting line at radial locations, by summing the velocity induced by each horseshoe vortex as follows:

$$
\begin{aligned}
& u_{a}^{*}(m)=\sum_{i=1}^{M} \bar{u}_{a}^{*}(m, i) \Gamma(i) \\
& u_{t}^{*}(m)=\sum_{i=1}^{M} \bar{u}_{t}^{*}(m, i) \Gamma(i)
\end{aligned}
$$

where $\bar{u}_{a}^{*}$ and $\bar{u}_{t}^{*}$ are the axial and tangential velocity induced at the certain radial location by a unit-strength horseshoe vortex surrounding panel $i$.

Lifting line theory does not take into account the induced velocities. Therefore, the vortice lattice theory is used for the calculation of the induced velocities, $\bar{u}_{a}^{*}$ and $\bar{u}_{t}^{*}$ as follows:

$$
\begin{aligned}
& \bar{u}_{a}^{*}(m, i)=\bar{u}_{a}(m, i)-\bar{u}_{a}(m, i+1) \\
& \bar{u}_{t}^{*}(m, i)=\bar{u}_{t}(m, i)-\bar{u}_{t}(m, i+1)
\end{aligned}
$$

where $\bar{u}_{a}(m, i)$ and $\bar{u}_{t}(m, i)$ are axial and tangential velocities induced at the certain radial locations by a unit-strength helical vortex filament at the panel end point with the vector direction of the circulation approaching the lifting line by right-hand rule. These components are calculated using formulae by Wrench (1957), [18].

More details regarding the lifting line theory can be found within $[18,19]$.

\subsection{Viscous flow theory}

In this subsection, basic physical laws for the description of the incompressible viscous flow along with the equations for Moving Reference Frame (MRF) method are given.

The law of conservation of mass in differential form is defined as [20]: 


$$
\frac{\partial \bar{u}_{i}}{\partial x_{i}}=0
$$

Reynolds-Averaged Navier Stokes equations for incompressible flow are given as [20]:

$$
\frac{\partial \bar{u}_{i}}{\partial t}+\frac{\partial}{\partial x_{j}}\left(\bar{u}_{i} \bar{u}_{j}+\overline{u_{i}^{\prime} u_{j}^{\prime}}\right)=-\frac{1}{\rho} \frac{\partial \bar{p}}{\partial x_{i}}+\frac{1}{\rho} \frac{\partial \bar{\tau}_{i j}}{\partial x_{j}}
$$

where $\bar{u}_{i}$ is the time averaged velocity vector, $\overline{u_{i}^{\prime} u_{j}^{\prime}}$ is the Reynolds stress tensor divided with $\rho, \bar{p}$ is the time averaged pressure and $\bar{\tau}_{i j}$ is defined as follows:

$$
\bar{\tau}_{i j}=\mu\left(\frac{\partial \bar{u}_{i}}{\partial x_{j}}+\frac{\partial \bar{u}_{j}}{\partial x_{i}}\right)
$$

In order to close an unclosed set of equations (10) and (11), Shear Stress Transport $k-\omega$ (SSTKO) turbulence model is introduced. This model includes modification for the influence of low Reynolds numbers. SSTKO is an empirical model, where one equation includes turbulent kinetic energy $(k)$ representing the velocity scale, and the other equation takes into account the dissipation rate $(\omega)$ representing the length scale.

Moving Reference Frame (MRF) method is utilized within numerical simulations of OWT, which are performed as steady simulations. OWT is carried out for each advance coefficient $(J)$, whereby the rate of revolution of a screw propeller is kept constant and advance speed is varied. In the case of MRF, the governing equations are solved together with additional acceleration terms. The computational domain is divided into stationary and rotational part. From the stationary point of view, the absolute velocity and the relative velocity are related with the equation:

$$
u_{0 i}=u_{p i}+\varepsilon_{i j k} \omega_{p j} r_{k}
$$

where $\omega_{p j}$ is the angular velocity and $r_{k}$ is the position vector from the origin of the MRF to the center of a control volume.

The law of conservation of mass and RANS equations in MRF written with relative velocity are as follows $[21,22]$ :

$$
\begin{aligned}
& \frac{\partial u_{p i}}{\partial x_{i}}=0 \\
& \frac{\partial u_{p i}}{\partial t}+u_{j} \frac{\partial u_{p i}}{\partial x_{j}}+\varepsilon_{i j k} \frac{\mathrm{d} \omega_{p j}}{\mathrm{~d} t} r_{k}+2 \varepsilon_{i j k} \omega_{p j} u_{p k}+\varepsilon_{i j k} \varepsilon_{j l m} \omega_{p l} \omega_{p m} r_{k}=-\frac{1}{\rho} \frac{\partial p}{\partial x_{i}}+v \frac{\partial^{2} u_{p i}}{\partial x_{j} \partial x_{j}}
\end{aligned}
$$

\section{Numerical setup}

In this section numerical setup used within numerical simulations of potential and viscous flow around a screw propeller is presented. 


\subsection{Lifting line theory}

Numerical simulations of potential flow are performed within open source code, OpenProp. Input data for the simulation are: profiles, chord length and pitch to diameter ratio of a screw propeller at different radii and maximum thickness distribution along the blade. In Figure 2, three-dimensional representation of the Gawn series propeller obtained from OpenProp is shown.

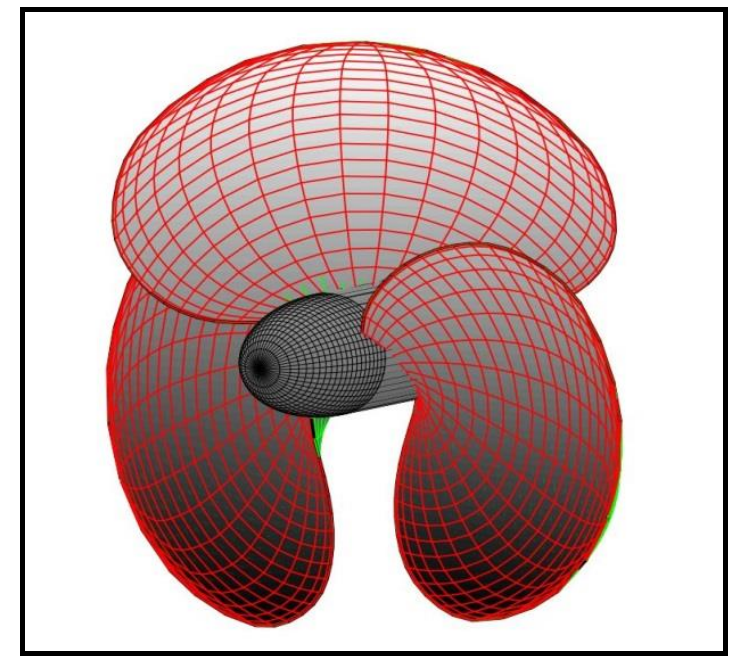

Fig. 2 3D representation of the Gawn series propeller

Geometry of Gawn series propeller is taken from [23]. Gawn series propellers have a uniform face pitch, segmental blade sections, constant blade thickness ratio and zero skewness. Within OpenProp, a text file is created which contains the position of the segmental blade sections in 3D in nondimensional form normalized with $D$. The shape of the hub is assumed to be cylindrical with diameter equal to $0.2 \mathrm{D}$. Text files are created for five propellers with the same expanded area ratio equal to 1.1 and with pitch to diameter ratio in the range from 0.7 to 0.9 with step of 0.05 . Numerical simulations are performed for a range of advance coefficients from 0.1 up to advance coefficient for which the thrust is negative. It should be noted that at higher $P / D$ ratios, i.e. for 0.85 and 0.9 , at lower advance coefficients screw propeller is more loaded. Therefore, for these two propellers, numerical simulations are performed from $J=0.15$ and $J=0.2$ respectively, since within OpenProp moderately loaded lifting line approach is used. Equations (1) and (2) are solved in a discrete form within OpenProp.

\subsection{Viscous flow theory}

Numerical simulations of viscous flow are performed within commercial software package, STAR-CCM+. Governing equations are discretized using finite volume method, which are solved in a segregated manner. All numerical simulations are performed as steady simulations for several advance coefficients in range from 0.1 to 0.75 with a step of 0.05 . The change of advance coefficient is achieved by variation of the inlet velocity, while rate of revolution is kept constant. The simulations for each advance coefficient are stopped after 4000 iterations when residuals drop at least four order of magnitude, i.e. iteration uncertainty is negligible, Figure 3. The computational domain, shown in Figure 4, is discretized using 
unstructured hexahedral mesh with following meshing tools: Prism layer mesher, Trimmer and Surface remesher according to the recommendations from [9].

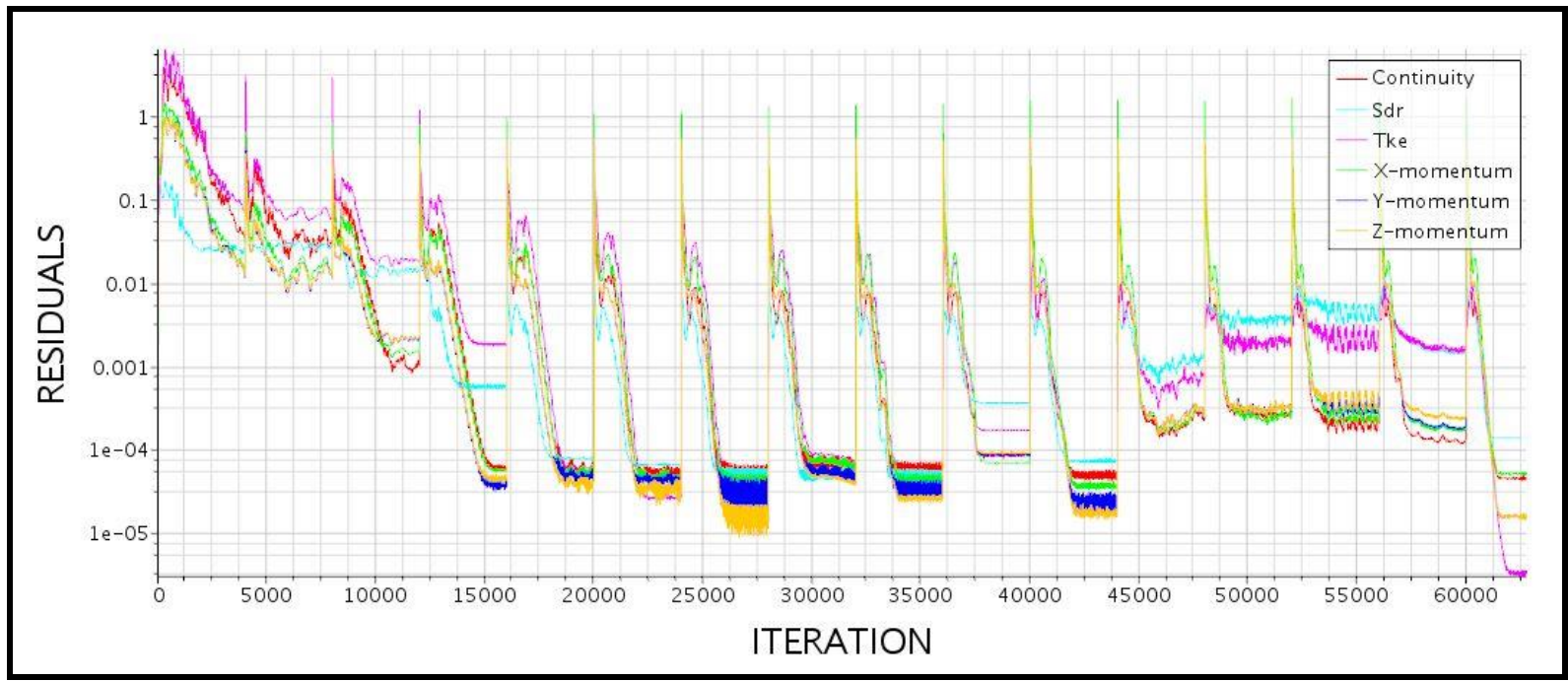

Fig. 3 Residuals plot

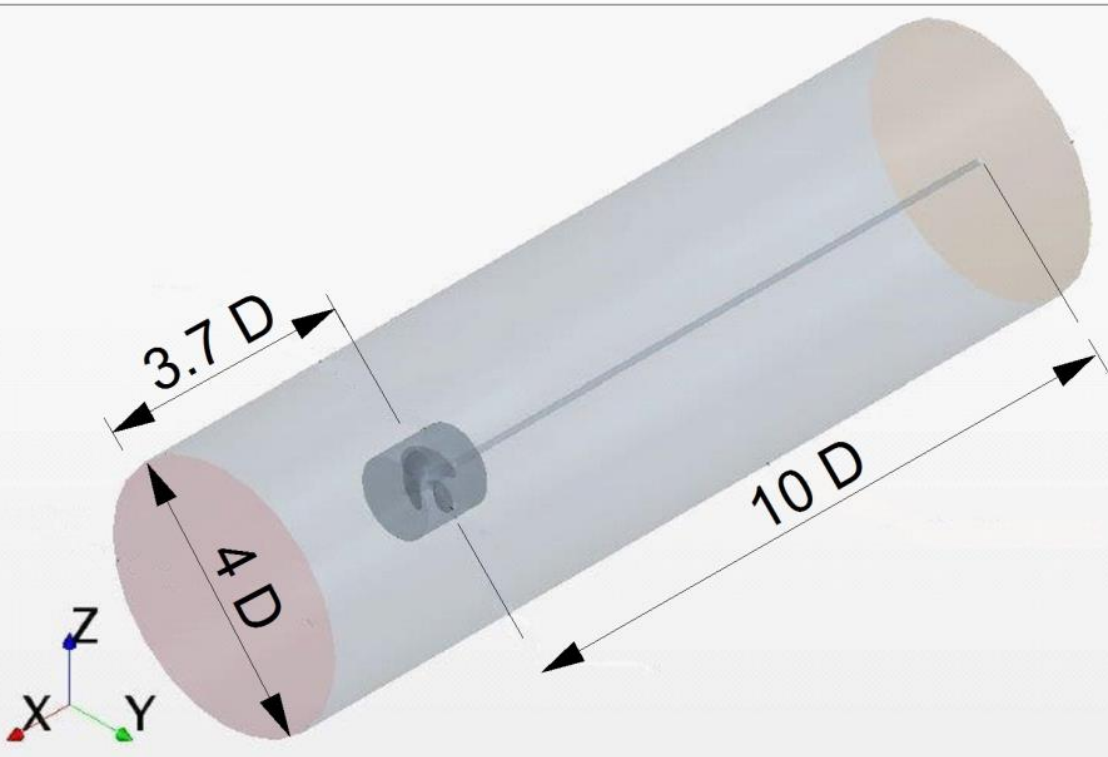

Fig. 4 Computational domain

Numerical simulations are performed for five Gawn series propellers of same expanded area ratio equal to 1.1 , which differ in pitch to diameter ratio ranging from 0.7 to 0.9 . The mesh for all five propellers has around 2.5 million cells. The mesh is refined through entire domain within the diameter equal to $1.4 \mathrm{D}$, and vertically near the propeller in the region equal to $1.4 \mathrm{D}$, as can be seen in Figure 5. The refinement in rotation region can be seen in Figure 6. 


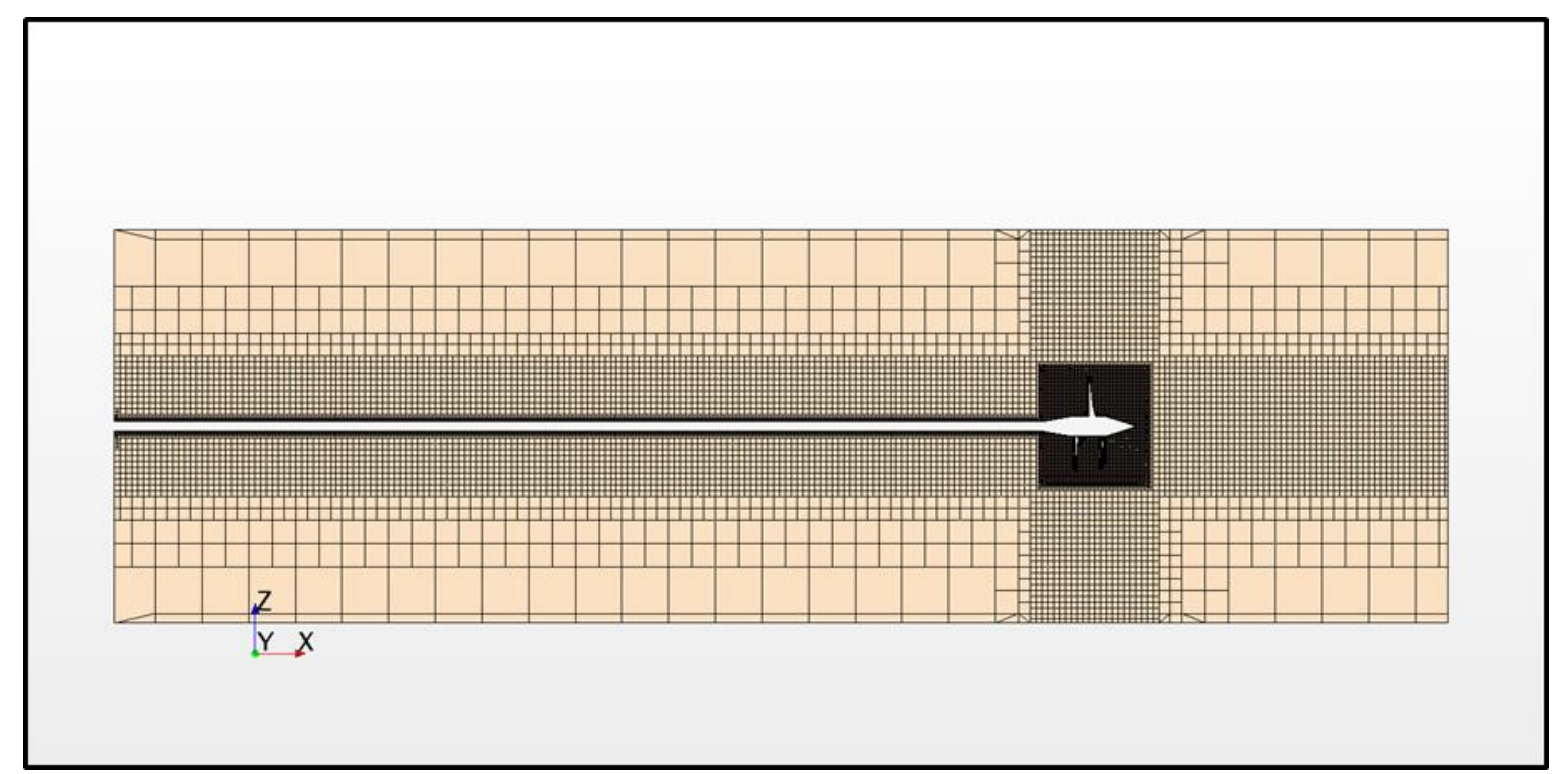

Fig. 5 Mesh refinements

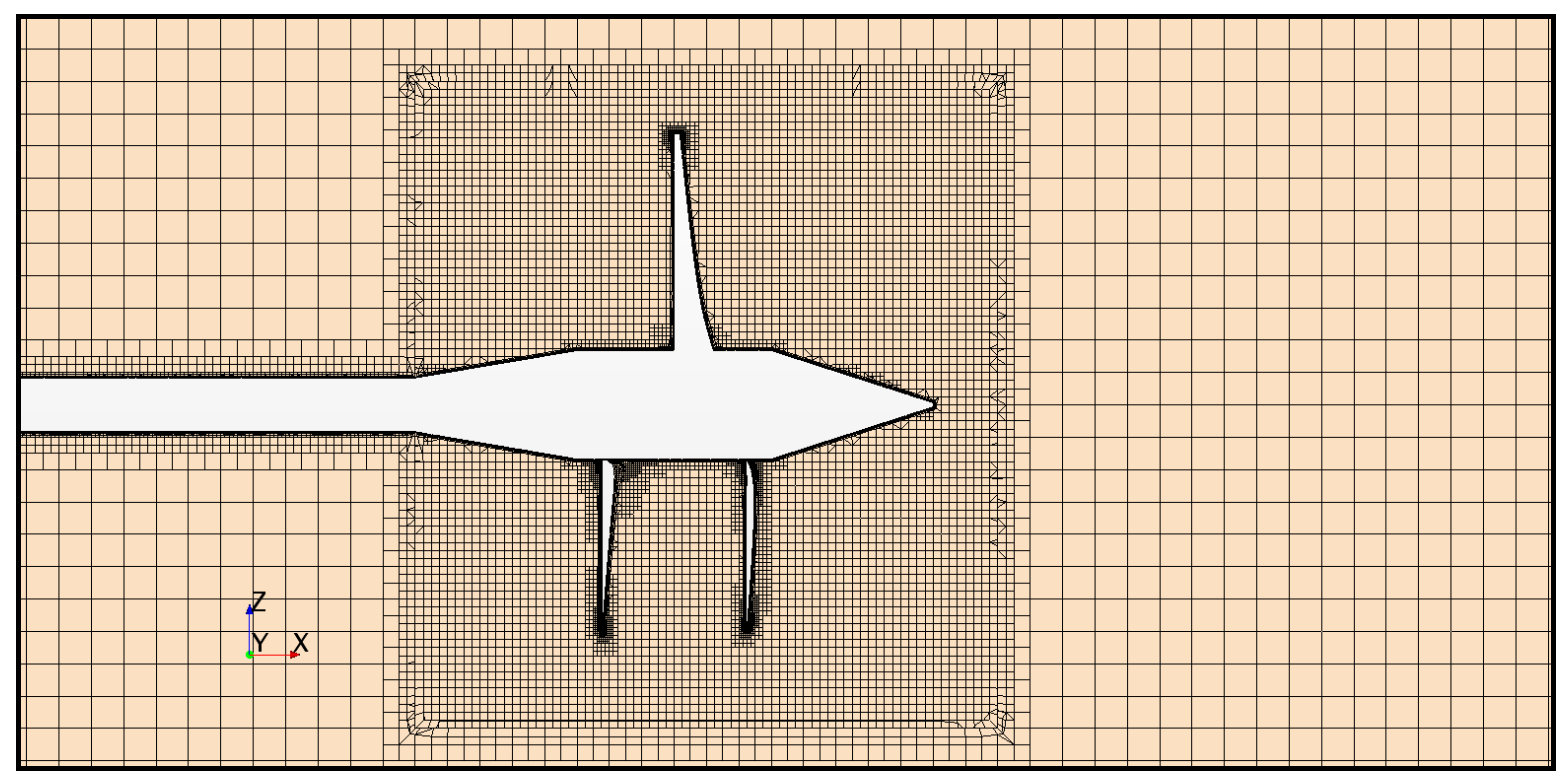

Fig. 6 Mesh refinement of rotation region

The special care is given to near wall treatment, where near wall cells are generated taking into account that $y^{+}$of the first cell near wall has value above 30, since wall functions are applied, Figure 7. It is important to note that underrelaxation factor for velocity is set to 0.5 and for pressure to 0.1 .

The boundary conditions are applied as follows: velocity inlet for inlet boundary, pressure outlet for outlet boundary, slip wall for lateral surface of the cylinder and no slip wall for the propeller. 


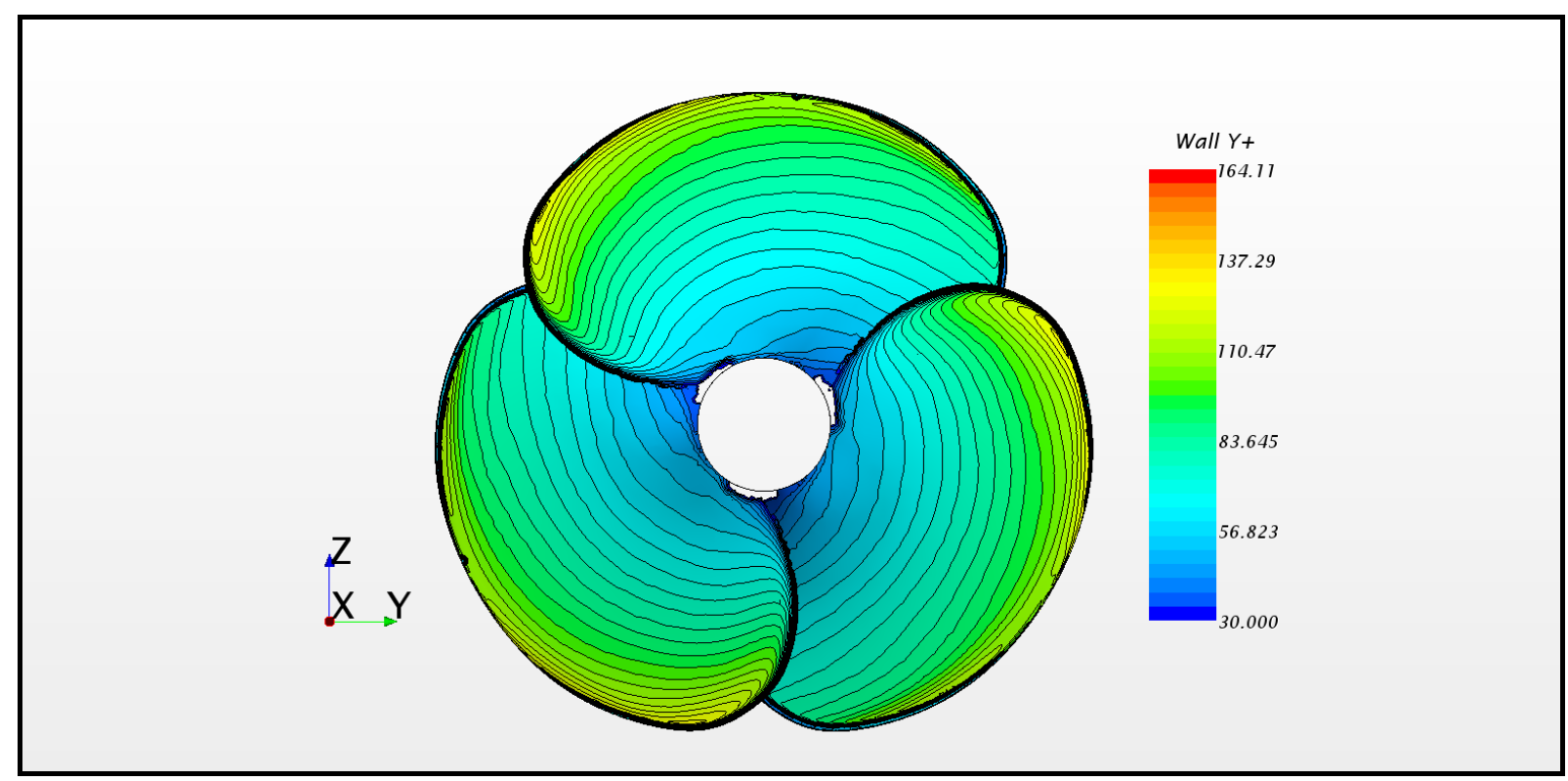

Fig. 7 The obtained $y^{+}$values at the first cell near wall

\section{Results}

Within this section, the results of OWT obtained utilizing lifting line method and RANS equations are presented. Furthermore, the viscous flow around the screw propeller in OWT is analysed. The obtained numerical hydrodynamic coefficients in OWT are compared with regression polynomials presented in [23], which are obtained from the experimental measurements as follows:

$$
\begin{aligned}
& K_{T}=\sum_{n=1}^{39} C_{n} \cdot J^{s} \cdot(P / D)^{t} \cdot\left(A_{E} / A_{0}\right)^{u} \cdot Z^{v} \\
& K_{Q}=\sum_{n=1}^{47} C_{n} \cdot J^{s} \cdot(P / D)^{t} \cdot\left(A_{E} / A_{0}\right)^{u} \cdot Z^{v}
\end{aligned}
$$

where $C_{n}, s, t, u, v$ are coefficients given in [23], $K_{T}$ is the thrust coefficient, $P / D$ is the pitch to diameter ratio, $A_{E} / A_{0}$ is the expanded area ratio and $K_{Q}$ is the torque coefficient.

The open water efficiency can be calculated as follows:

$$
\eta_{\mathrm{O}}=\frac{J}{2 \pi} \frac{K_{T}}{K_{Q}}
$$

OWT diagrams obtained utilizing lifting line theory and RANS simulations are compared with regression polynomials, Figures 8-12. It can be noticed that satisfactory agreement between numerical results obtained using viscous flow theory and regression polynomials is achieved. Differences in $K_{T}$ and $10 K_{Q}$ obtained using RANS and with regression polynomials are relatively low. However, as results for $K_{T}$ obtained with RANS underestimate the solution obtained by regression polynomials and results for $10 K_{Q}$ obtained with RANS overestimate the solution obtained by regression polynomials, the obtained 
differences in $\eta_{\mathrm{O}}$ are even more pronounced. Results of numerical simulations based on the lifting line theory have larger deviations from regression polynomials for thrust and torque coefficients at lower values of $J$. The reason for this is that propeller is more loaded at lower $J$ values. Mathematical model in OpenProp uses an assumption of moderately loaded propeller blade and thus the radial component of induced velocity is neglected. However, heavily loaded propellers have significant value of induced radial velocity and for that reason OpenProp can be used for simulations in the range of $J$ where the propeller is moderately loaded. Trends of hydrodynamic coefficients obtained with RANS simulations are the same as the ones obtained using regression polynomials, while the ones obtained with lifting line theory show significant deviations at lower $J$ values. However, in the range of $J$ where propeller is moderately loaded, trends of hydrodynamic coefficients obtained with numerical simulations based on lifting line theory and regression polynomials are the same. As the most of screw propellers operate in the range of $J$ where propeller is moderately loaded, it can be concluded that OpenProp can be used for preliminary design of a screw propeller.

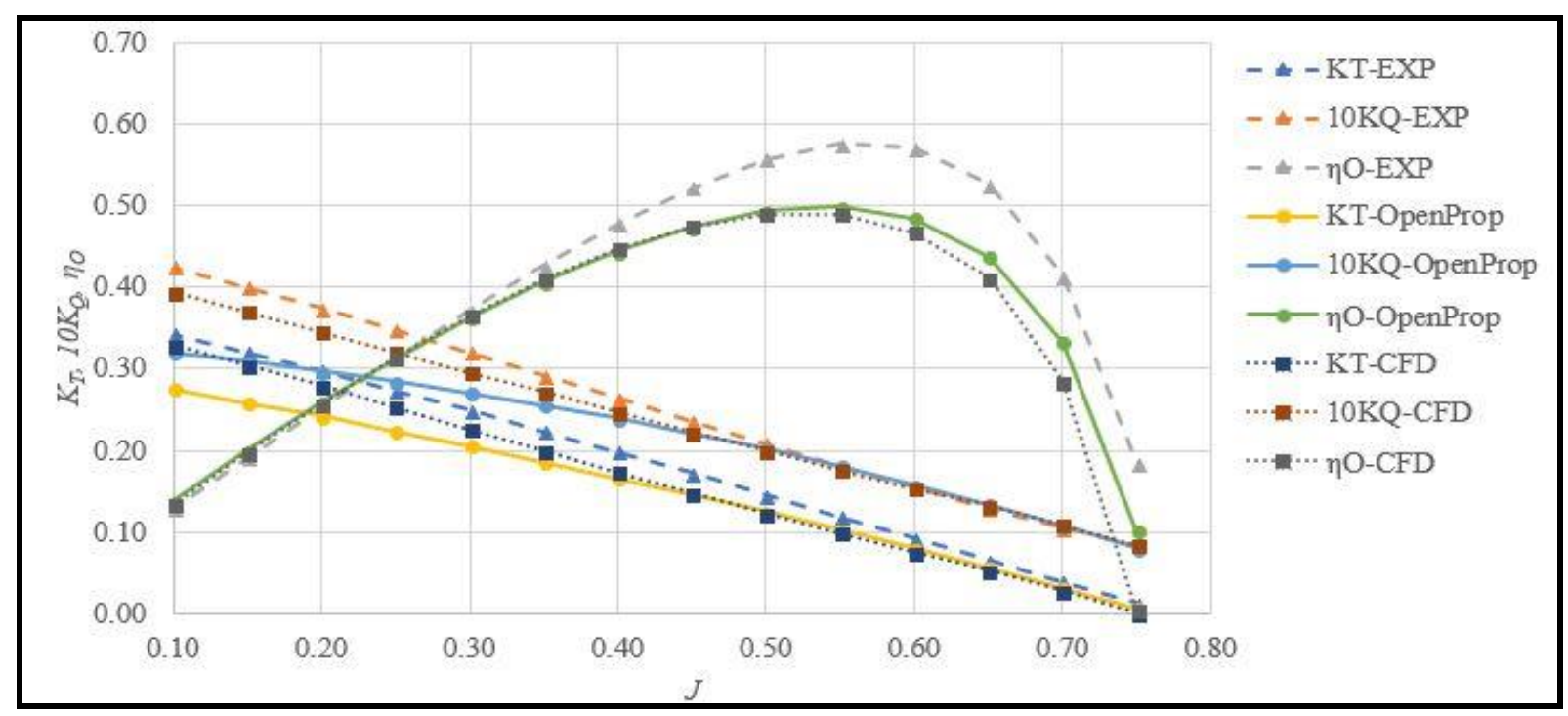

Fig. 8 OWT diagram for $P / D=0.7$

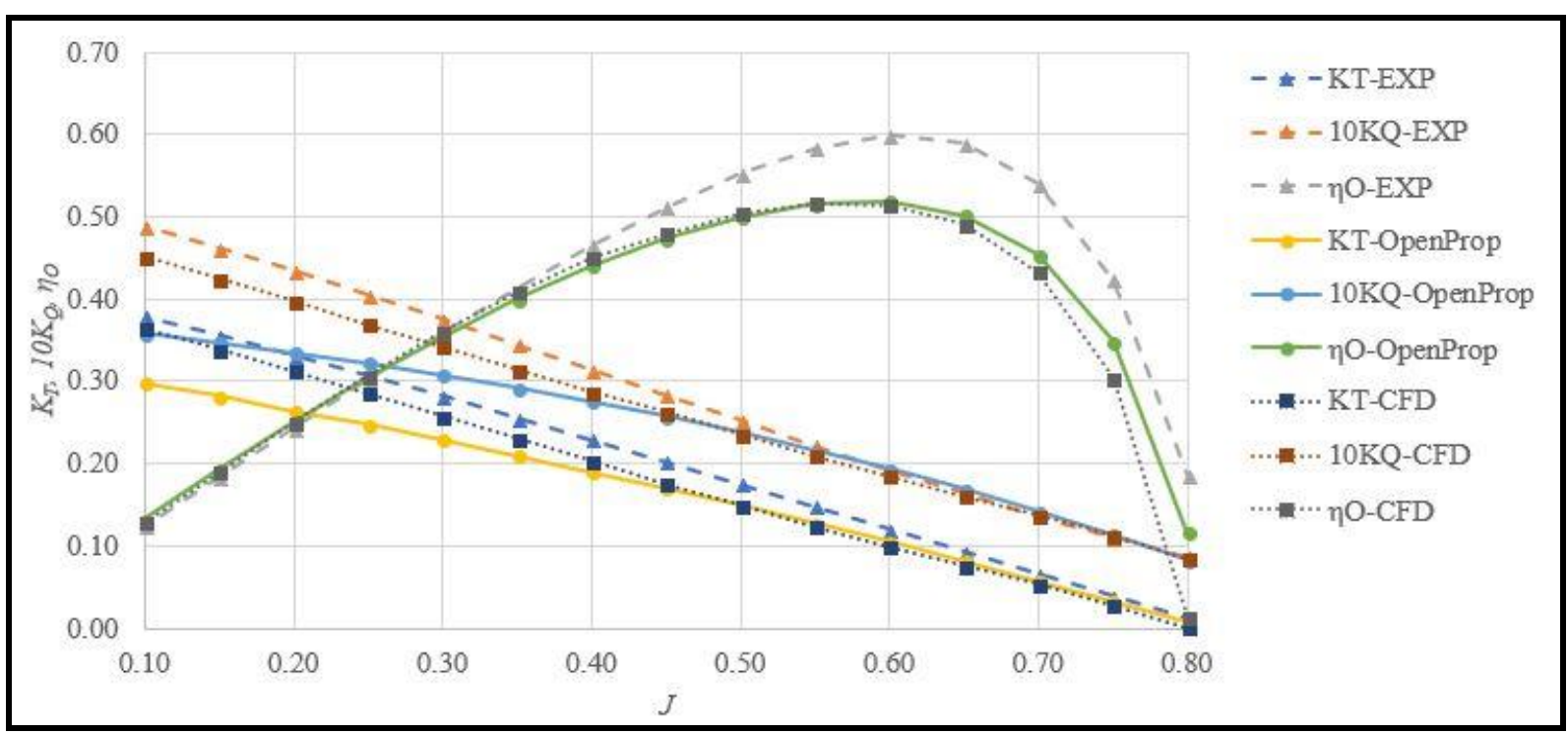

Fig. 9 OWT diagram for $P / D=0.75$ 


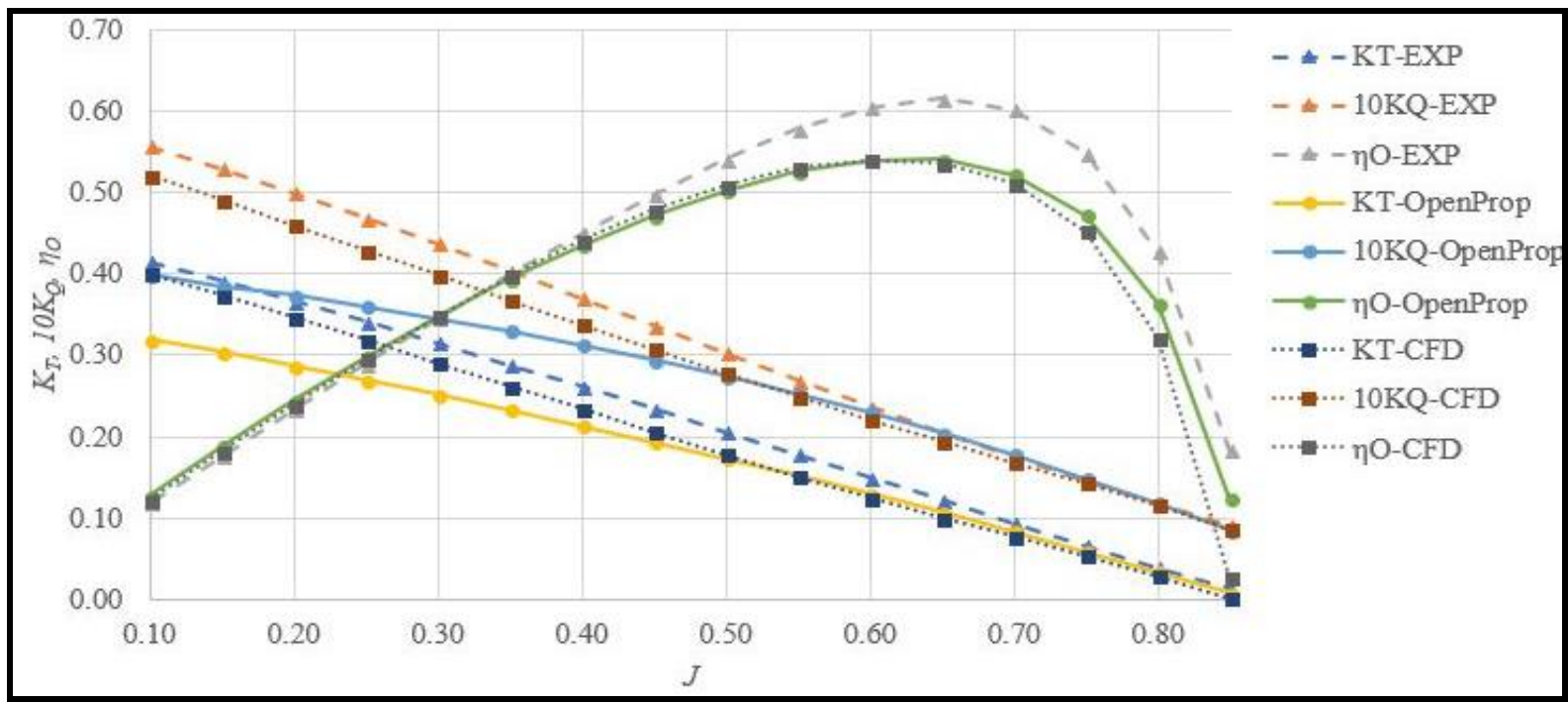

Fig. 10 OWT diagram for $P / D=0.8$

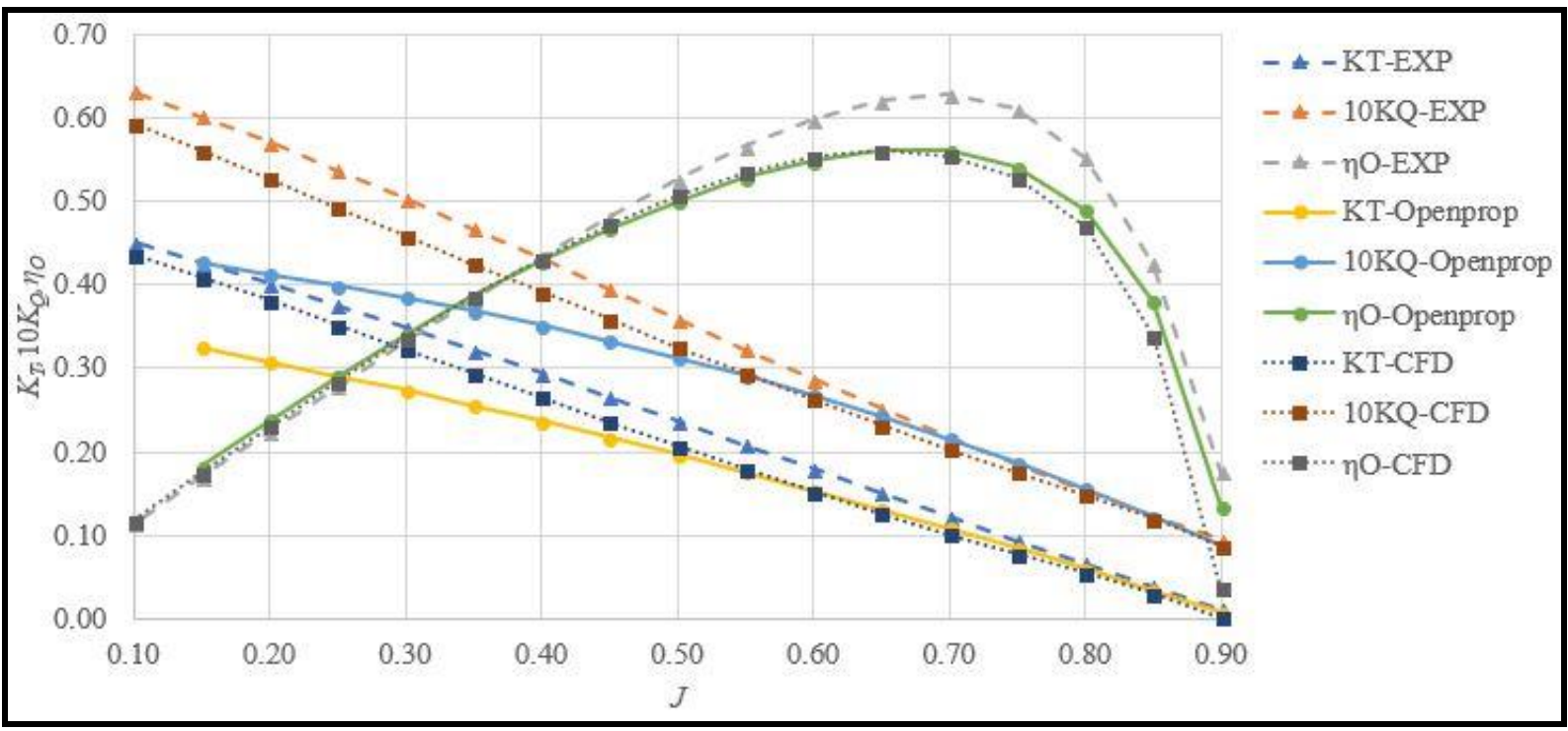

Fig. 11 OWT diagram for $P / D=0.85$

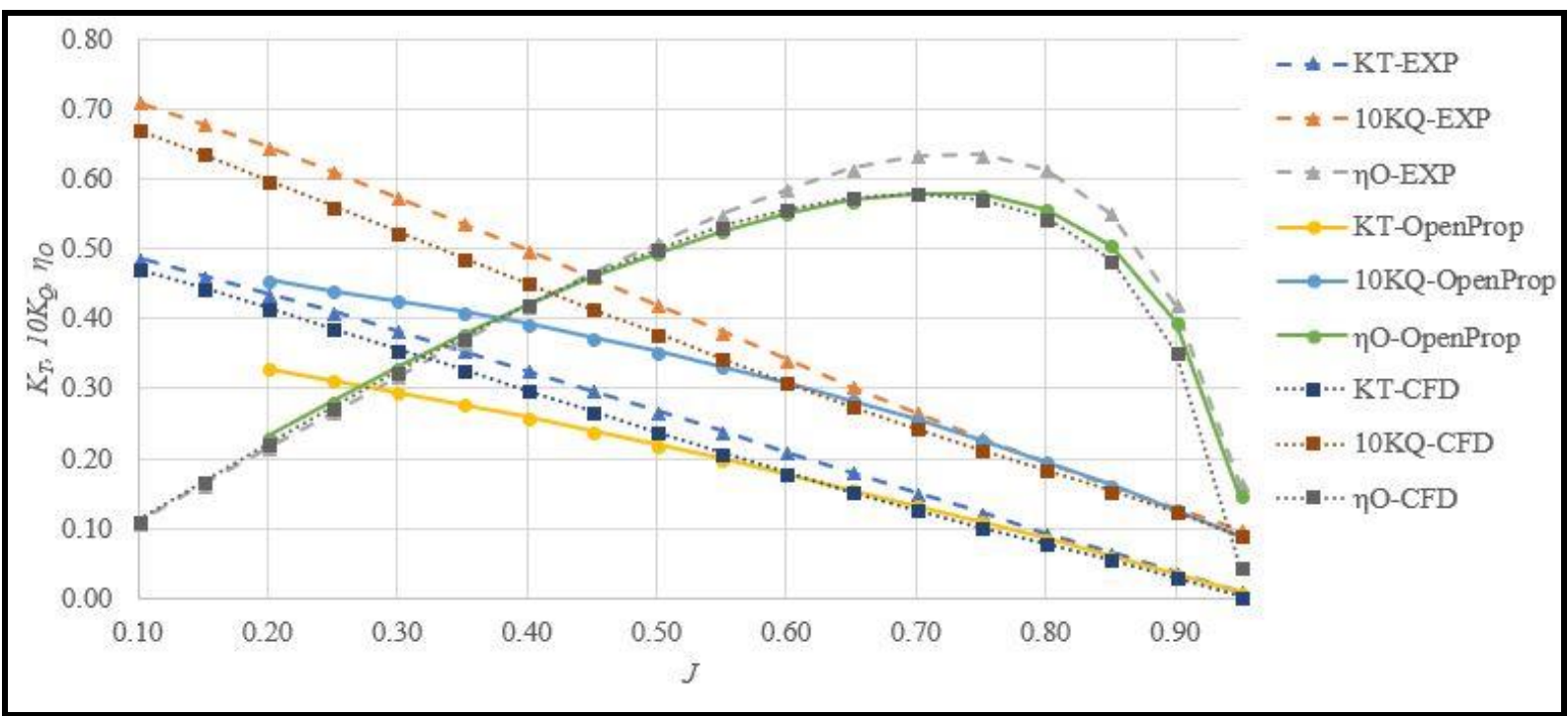

Fig.12 OWT diagram for $P / D=0.9$ 
Tables 1-5 show results of OWT obtained by OpenProp (potential flow), STAR CCM+ (RANS) and regression polynomials for different pitch to diameter ratio.

Table 1: OWT results for $\mathrm{P} / \mathrm{D}=0.7$

\begin{tabular}{|c|c|c|c|c|c|c|c|c|c|}
\hline$J$ & $K_{T}$-EXP & $K_{T}$-CFD & $K_{T}$-OpenProp & $10 K_{Q}$-EXP & $10 K_{Q}$-CFD & $10 K_{Q}$-OpenProp & $\eta_{\mathrm{O}}$-EXP & $\eta_{\mathrm{O}^{-}}$CFD & $\eta_{\mathrm{O}}$-OpenProp \\
\hline 0.10 & 0.3428 & 0.3287 & 0.2753 & 0.4242 & 0.3930 & 0.3198 & 0.129 & 0.133 & 0.137 \\
\hline 0.15 & 0.3206 & 0.3044 & 0.2585 & 0.3999 & 0.3695 & 0.3088 & 0.191 & 0.197 & 0.200 \\
\hline 0.20 & 0.2974 & 0.2787 & 0.2414 & 0.3743 & 0.3449 & 0.2969 & 0.253 & 0.257 & 0.259 \\
\hline 0.25 & 0.2735 & 0.2525 & 0.2235 & 0.3475 & 0.3202 & 0.2839 & 0.313 & 0.314 & 0.313 \\
\hline 0.30 & 0.2488 & 0.2261 & 0.2052 & 0.3200 & 0.2957 & 0.2701 & 0.371 & 0.365 & 0.363 \\
\hline 0.35 & 0.2235 & 0.1996 & 0.1862 & 0.2920 & 0.2711 & 0.2551 & 0.426 & 0.410 & 0.407 \\
\hline 0.40 & 0.1978 & 0.1732 & 0.1665 & 0.2638 & 0.2467 & 0.2387 & 0.477 & 0.447 & 0.444 \\
\hline 0.45 & 0.1717 & 0.1473 & 0.1460 & 0.2356 & 0.2224 & 0.2208 & 0.522 & 0.474 & 0.473 \\
\hline 0.50 & 0.1453 & 0.1224 & 0.1247 & 0.2077 & 0.1989 & 0.2014 & 0.557 & 0.490 & 0.493 \\
\hline 0.55 & 0.1187 & 0.0984 & 0.1027 & 0.1804 & 0.1761 & 0.1804 & 0.576 & 0.489 & 0.498 \\
\hline 0.60 & 0.0921 & 0.0753 & 0.0799 & 0.1541 & 0.1538 & 0.1578 & 0.571 & 0.468 & 0.484 \\
\hline 0.65 & 0.0655 & 0.0524 & 0.0564 & 0.1289 & 0.1319 & 0.1334 & 0.525 & 0.411 & 0.437 \\
\hline 0.70 & 0.0390 & 0.0277 & 0.0320 & 0.1052 & 0.1088 & 0.1073 & 0.413 & 0.283 & 0.332 \\
\hline 0.75 & 0.0128 & 0.0003 & 0.0067 & 0.0832 & 0.0833 & 0.0794 & 0.184 & 0.004 & 0.101 \\
\hline
\end{tabular}

Table 2: $\mathrm{OWT}$ results for $\mathrm{P} / \mathrm{D}=0.75$

\begin{tabular}{|c|c|c|c|c|c|c|c|c|c|}
\hline$J$ & $K_{T}$-EXP & $K_{T}$-CFD & $K_{T}$-OpenProp & $10 K_{Q}$-EXP & $10 K_{Q}$-CFD & $10 K_{Q}$-OpenProp & $\eta_{\mathrm{O}}$-EXP & $\eta_{\mathrm{O}}$-CFD & $\eta_{\mathrm{O}}$-OpenProp \\
\hline 0.10 & 0.3786 & 0.3645 & 0.2983 & 0.4881 & 0.4515 & 0.3579 & 0.123 & 0.128 & 0.133 \\
\hline 0.15 & 0.3556 & 0.3391 & 0.2816 & 0.4619 & 0.4248 & 0.3473 & 0.184 & 0.191 & 0.194 \\
\hline 0.20 & 0.3318 & 0.3124 & 0.2642 & 0.4342 & 0.3971 & 0.3349 & 0.243 & 0.250 & 0.251 \\
\hline 0.25 & 0.3071 & 0.2852 & 0.2473 & 0.4054 & 0.3693 & 0.3221 & 0.301 & 0.307 & 0.305 \\
\hline 0.30 & 0.2817 & 0.2580 & 0.2289 & 0.3756 & 0.3418 & 0.3077 & 0.358 & 0.360 & 0.355 \\
\hline 0.35 & 0.2558 & 0.2306 & 0.2101 & 0.3451 & 0.3145 & 0.2924 & 0.413 & 0.408 & 0.400 \\
\hline 0.40 & 0.2293 & 0.2032 & 0.1905 & 0.3142 & 0.2872 & 0.2756 & 0.465 & 0.450 & 0.440 \\
\hline 0.45 & 0.2025 & 0.1758 & 0.1703 & 0.2833 & 0.2625 & 0.2575 & 0.512 & 0.480 & 0.474 \\
\hline 0.50 & 0.1753 & 0.1493 & 0.1493 & 0.2525 & 0.2359 & 0.2378 & 0.553 & 0.504 & 0.500 \\
\hline 0.55 & 0.1481 & 0.1239 & 0.1276 & 0.2222 & 0.2101 & 0.2165 & 0.583 & 0.516 & 0.516 \\
\hline 0.60 & 0.1207 & 0.0996 & 0.1052 & 0.1926 & 0.1852 & 0.1935 & 0.599 & 0.514 & 0.519 \\
\hline 0.65 & 0.0934 & 0.0764 & 0.0819 & 0.1639 & 0.1611 & 0.1687 & 0.589 & 0.490 & 0.503 \\
\hline 0.70 & 0.0662 & 0.0533 & 0.0580 & 0.1366 & 0.1373 & 0.1422 & 0.540 & 0.433 & 0.455 \\
\hline 0.75 & 0.0393 & 0.0286 & 0.0332 & 0.1108 & 0.1123 & 0.1138 & 0.423 & 0.304 & 0.349 \\
\hline 0.80 & 0.0127 & 0.0010 & 0.0076 & 0.0869 & 0.0845 & 0.0836 & 0.187 & 0.015 & 0.116 \\
\hline
\end{tabular}


Table 3: OWT results for $\mathrm{P} / \mathrm{D}=0.8$

\begin{tabular}{|c|c|c|c|c|c|c|c|c|c|}
\hline$J$ & $K_{T}$-EXP & $K_{T}$-CFD & $K_{T}$-OpenProp & $10 K_{Q}$-EXP & $10 K_{Q}$-CFD & $10 K_{Q}$-OpenProp & $\eta_{\mathrm{O}}$-EXP & $\eta_{\mathrm{O}}$-CFD & $\eta_{\mathrm{O}}$-OpenProp \\
\hline 0.10 & 0.4145 & 0.4000 & 0.3189 & 0.5572 & 0.5206 & 0.3980 & 0.118 & 0.122 & 0.128 \\
\hline 0.15 & 0.3909 & 0.3738 & 0.3035 & 0.5292 & 0.4909 & 0.3854 & 0.176 & 0.182 & 0.188 \\
\hline 0.20 & 0.3664 & 0.3463 & 0.2867 & 0.4996 & 0.4599 & 0.3735 & 0.233 & 0.240 & 0.244 \\
\hline 0.25 & 0.3411 & 0.3183 & 0.2692 & 0.4686 & 0.4288 & 0.3597 & 0.290 & 0.295 & 0.298 \\
\hline 0.30 & 0.3150 & 0.2902 & 0.2516 & 0.4365 & 0.3983 & 0.3455 & 0.345 & 0.348 & 0.348 \\
\hline 0.35 & 0.2884 & 0.2620 & 0.2330 & 0.4037 & 0.3679 & 0.3297 & 0.398 & 0.397 & 0.394 \\
\hline 0.40 & 0.2613 & 0.2337 & 0.2137 & 0.3703 & 0.3377 & 0.3128 & 0.449 & 0.441 & 0.435 \\
\hline 0.45 & 0.2338 & 0.2056 & 0.1937 & 0.3366 & 0.3078 & 0.2944 & 0.497 & 0.478 & 0.471 \\
\hline 0.50 & 0.2060 & 0.1779 & 0.1731 & 0.3030 & 0.2783 & 0.2745 & 0.541 & 0.509 & 0.502 \\
\hline 0.55 & 0.1780 & 0.1509 & 0.1517 & 0.2697 & 0.2493 & 0.2529 & 0.578 & 0.530 & 0.525 \\
\hline 0.60 & 0.1499 & 0.1250 & 0.1299 & 0.2369 & 0.2212 & 0.2300 & 0.604 & 0.540 & 0.539 \\
\hline 0.65 & 0.1219 & 0.1006 & 0.1070 & 0.2049 & 0.1944 & 0.2048 & 0.615 & 0.535 & 0.540 \\
\hline 0.70 & 0.0940 & 0.0772 & 0.0833 & 0.1741 & 0.1685 & 0.1778 & 0.602 & 0.510 & 0.522 \\
\hline 0.75 & 0.0664 & 0.0542 & 0.0589 & 0.1446 & 0.1430 & 0.1490 & 0.548 & 0.452 & 0.472 \\
\hline 0.80 & 0.0391 & 0.0293 & 0.0337 & 0.1167 & 0.1161 & 0.1182 & 0.427 & 0.322 & 0.363 \\
\hline 0.85 & 0.0123 & 0.0017 & 0.0078 & 0.0908 & 0.0860 & 0.0856 & 0.183 & 0.026 & 0.123 \\
\hline
\end{tabular}

Table 4: OWT results for $\mathrm{P} / \mathrm{D}=0.85$

\begin{tabular}{|c|c|c|c|c|c|c|c|c|c|}
\hline$J$ & $K_{T}$-EXP & $K_{T}$-CFD & $K_{T}$-OpenProp & $10 K_{Q}$-EXP & $10 K_{Q}$-CFD & $10 K_{Q}$-OpenProp & $\eta_{\mathrm{O}}$-EXP & $\eta_{\mathrm{O}}$-CFD & $\eta_{\mathrm{O}}$-OpenProp \\
\hline 0.10 & 0.4506 & 0.4357 & $/$ & 0.6314 & 0.5930 & $/$ & 0.114 & 0.117 & $/$ \\
\hline 0.15 & 0.4264 & 0.4088 & 0.3250 & 0.6016 & 0.5604 & 0.4266 & 0.169 & 0.174 & 0.138 \\
\hline 0.20 & 0.4012 & 0.3806 & 0.3083 & 0.5701 & 0.5262 & 0.4128 & 0.224 & 0.230 & 0.186 \\
\hline 0.25 & 0.3753 & 0.3519 & 0.2908 & 0.5370 & 0.4919 & 0.3991 & 0.278 & 0.285 & 0.235 \\
\hline 0.30 & 0.3486 & 0.3231 & 0.2737 & 0.5028 & 0.4581 & 0.3846 & 0.331 & 0.337 & 0.285 \\
\hline 0.35 & 0.3214 & 0.2942 & 0.2556 & 0.4676 & 0.4247 & 0.3688 & 0.383 & 0.386 & 0.335 \\
\hline 0.40 & 0.2936 & 0.2651 & 0.2366 & 0.4317 & 0.3913 & 0.3516 & 0.433 & 0.431 & 0.385 \\
\hline 0.45 & 0.2654 & 0.2361 & 0.2170 & 0.3954 & 0.3582 & 0.3332 & 0.481 & 0.472 & 0.434 \\
\hline 0.50 & 0.2370 & 0.2074 & 0.1966 & 0.3590 & 0.3258 & 0.3131 & 0.525 & 0.507 & 0.480 \\
\hline 0.55 & 0.2083 & 0.1793 & 0.1756 & 0.3227 & 0.2938 & 0.2914 & 0.565 & 0.534 & 0.523 \\
\hline 0.60 & 0.1796 & 0.1519 & 0.1539 & 0.2868 & 0.2624 & 0.2680 & 0.598 & 0.553 & 0.560 \\
\hline 0.65 & 0.1509 & 0.1257 & 0.1314 & 0.2516 & 0.2320 & 0.2427 & 0.621 & 0.560 & 0.586 \\
\hline 0.70 & 0.1224 & 0.1012 & 0.1085 & 0.2173 & 0.2036 & 0.2159 & 0.627 & 0.554 & 0.594 \\
\hline 0.75 & 0.0941 & 0.0778 & 0.0846 & 0.1842 & 0.1759 & 0.1868 & 0.610 & 0.528 & 0.574 \\
\hline 0.80 & 0.0661 & 0.0548 & 0.0599 & 0.1526 & 0.1487 & 0.1557 & 0.551 & 0.469 & 0.513 \\
\hline 0.85 & 0.0386 & 0.0300 & 0.0344 & 0.1228 & 0.1198 & 0.1226 & 0.425 & 0.339 & 0.389 \\
\hline 0.90 & 0.0116 & 0.0023 & 0.0082 & 0.0949 & 0.0876 & 0.0876 & 0.176 & 0.037 & 0.135 \\
\hline
\end{tabular}


Table 5: OWT results for $\mathrm{P} / \mathrm{D}=0.9$

\begin{tabular}{|c|c|c|c|c|c|c|c|c|c|}
\hline$J$ & $K_{T}$-EXP & $K_{T}$-CFD & $K_{T}$-OpenProp & $10 K_{Q}$-EXP & $10 K_{Q}$-CFD & $10 K_{Q}$-OpenProp & $\eta_{\mathrm{O}}$-EXP & $\eta_{\mathrm{O}}$-CFD & $\eta_{\mathrm{O}}$-OpenProp \\
\hline 0.10 & 0.4866 & 0.4710 & $/$ & 0.7106 & 0.6707 & $/$ & 0.109 & 0.112 & $/$ \\
\hline 0.15 & 0.4618 & 0.4438 & $/$ & 0.6790 & 0.6355 & $/$ & 0.162 & 0.167 & $/$ \\
\hline 0.20 & 0.4361 & 0.4150 & 0.3291 & 0.6455 & 0.5984 & 0.4550 & 0.215 & 0.221 & 0.230 \\
\hline 0.25 & 0.4096 & 0.3857 & 0.3122 & 0.6105 & 0.5610 & 0.4405 & 0.267 & 0.274 & 0.282 \\
\hline 0.30 & 0.3823 & 0.3564 & 0.2947 & 0.5741 & 0.5240 & 0.4250 & 0.318 & 0.325 & 0.331 \\
\hline 0.35 & 0.3545 & 0.3269 & 0.2775 & 0.5366 & 0.4874 & 0.4096 & 0.368 & 0.374 & 0.377 \\
\hline 0.40 & 0.3261 & 0.2972 & 0.2590 & 0.4983 & 0.4508 & 0.3923 & 0.417 & 0.420 & 0.420 \\
\hline 0.45 & 0.2974 & 0.2674 & 0.2397 & 0.4595 & 0.4145 & 0.3736 & 0.464 & 0.462 & 0.459 \\
\hline 0.50 & 0.2683 & 0.2378 & 0.2198 & 0.4203 & 0.3787 & 0.3536 & 0.508 & 0.500 & 0.495 \\
\hline 0.55 & 0.2390 & 0.2088 & 0.1991 & 0.3812 & 0.3436 & 0.3318 & 0.549 & 0.532 & 0.525 \\
\hline 0.60 & 0.2097 & 0.1803 & 0.1778 & 0.3423 & 0.3092 & 0.3084 & 0.585 & 0.557 & 0.551 \\
\hline 0.65 & 0.1804 & 0.1526 & 0.1557 & 0.3039 & 0.2756 & 0.2830 & 0.614 & 0.573 & 0.569 \\
\hline 0.70 & 0.1512 & 0.1263 & 0.1334 & 0.2662 & 0.2432 & 0.2563 & 0.633 & 0.579 & 0.580 \\
\hline 0.75 & 0.1222 & 0.1018 & 0.1099 & 0.2297 & 0.2130 & 0.2270 & 0.635 & 0.570 & 0.578 \\
\hline 0.80 & 0.0936 & 0.0784 & 0.0857 & 0.1944 & 0.1837 & 0.1958 & 0.613 & 0.543 & 0.557 \\
\hline 0.85 & 0.0654 & 0.0553 & 0.0608 & 0.1607 & 0.1546 & 0.1626 & 0.551 & 0.484 & 0.506 \\
\hline 0.90 & 0.0378 & 0.0305 & 0.0352 & 0.1288 & 0.1239 & 0.1273 & 0.420 & 0.353 & 0.396 \\
\hline 0.95 & 0.0108 & 0.0027 & 0.0087 & 0.0990 & 0.0893 & 0.0899 & 0.165 & 0.046 & 0.147 \\
\hline
\end{tabular}

The pressure field on the pressure side of the propeller for two values of $J$ is shown in Figure 13 for propeller with $P / D=0.8$. It can be noticed that on the pressure side of the propeller for higher $J$, the overpressure is lower since the load on the propeller is lower. The pressure field on the suction side of the propeller with $P / D=0.8$ for two same values of $J$ is shown in Figure 14. For higher $J$, the underpressure on the suction side of the propeller is reduced. The reason for this is an increase in inflow velocity and decrease of the load on the propeller. Since approximately two-thirds of the thrust are caused by the underpressure on the suction side of propeller blades [1], the thrust is lower at higher values of $J$.
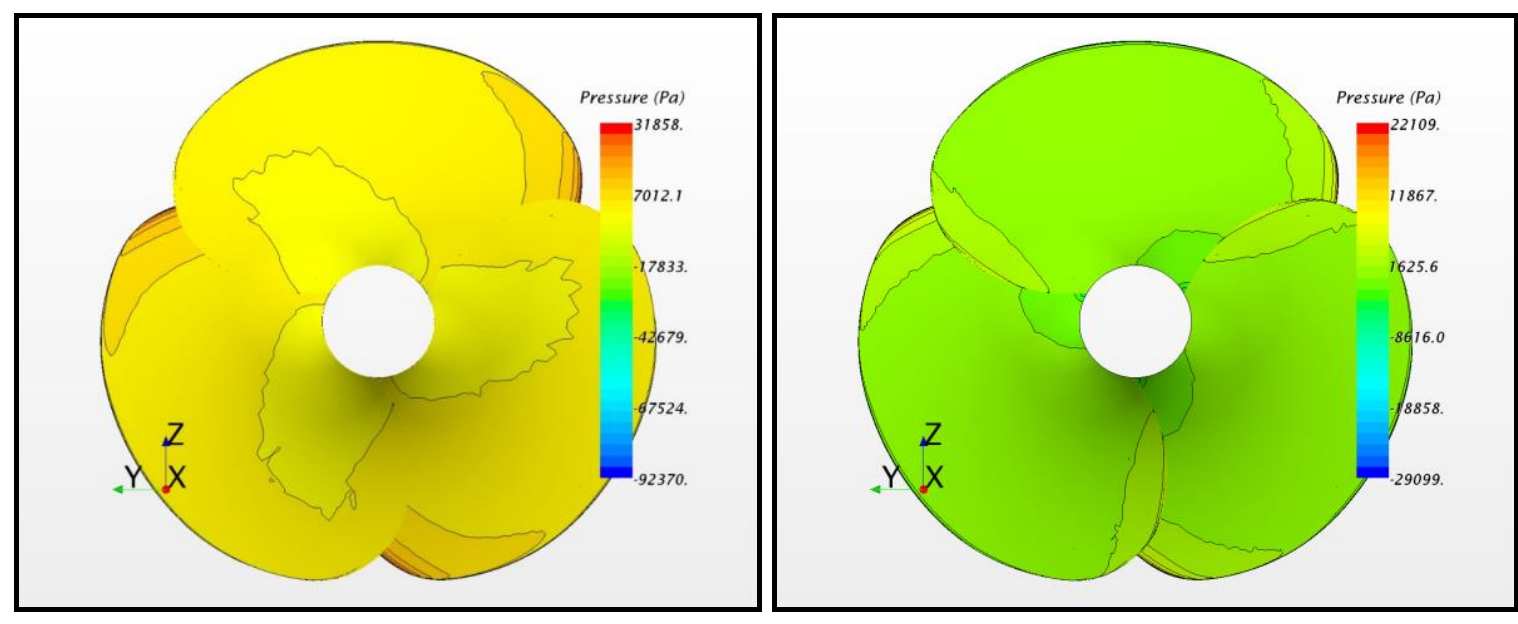

Fig. 13 Pressure distribution on the pressure side of the screw propeller at $J=0.2$ (left) and $J=0.55$ (right) 

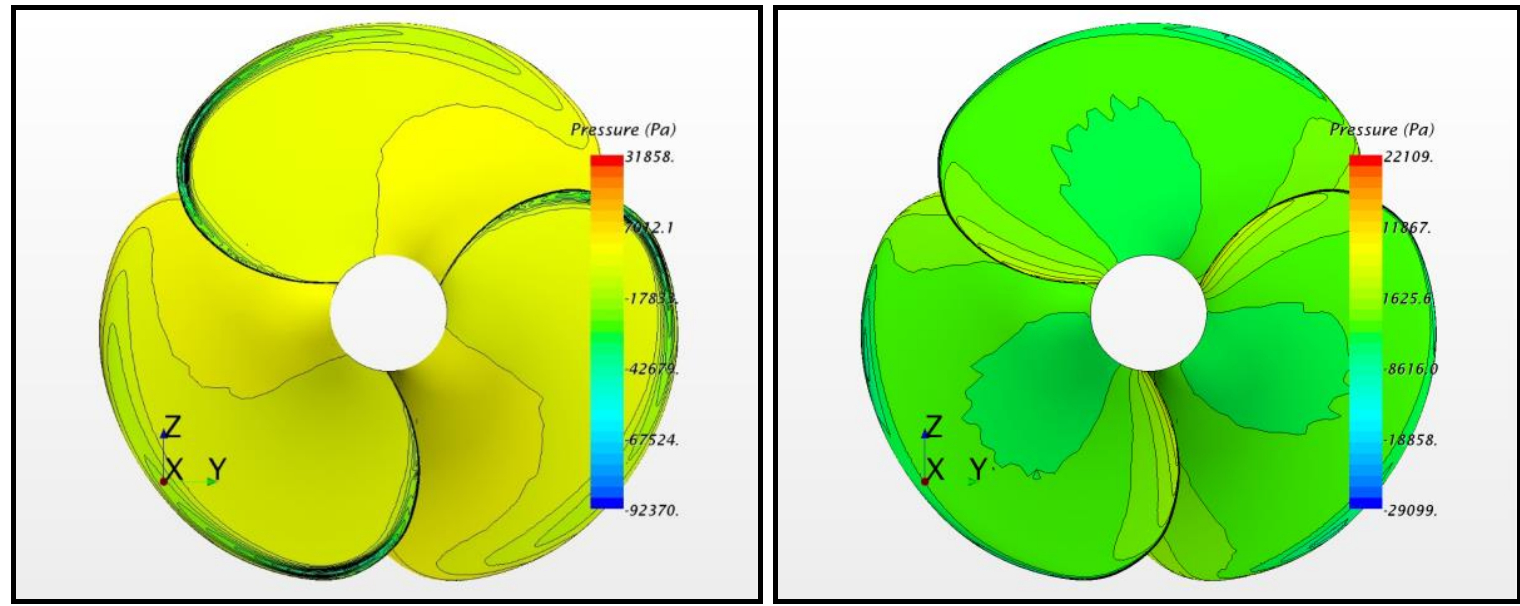

Fig. 14 Pressure distribution on the suction side of the screw propeller at $J=0.2$ (left) and $J=0.55$ (right)
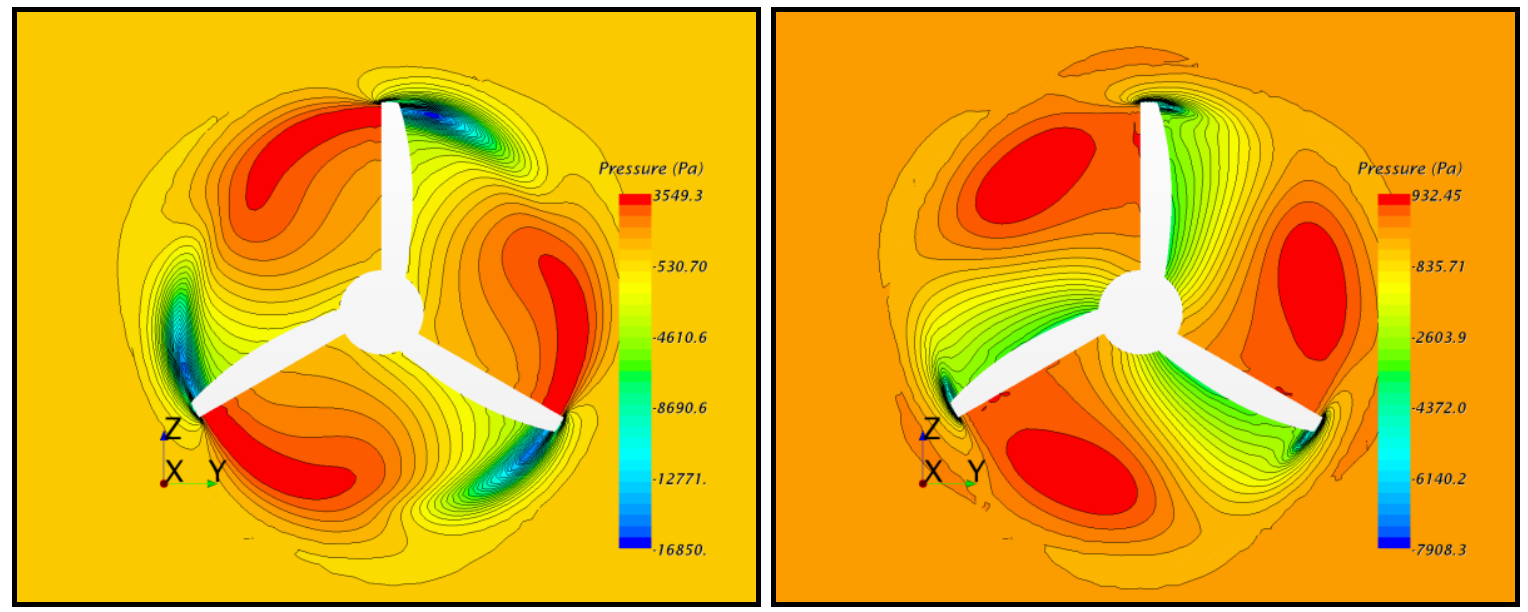

Fig. 15 Pressure distribution in the vertical plane at $J=0.2$ (left) and $J=0.55$ (right)

In Figure 15, the pressure field of propeller with $P / D=0.8$ in the vertical plane is shown. The larger difference in the pressure between the pressure and suction side of the propeller can be noticed at lower $J$ value, which is caused by larger load on the propeller blades.
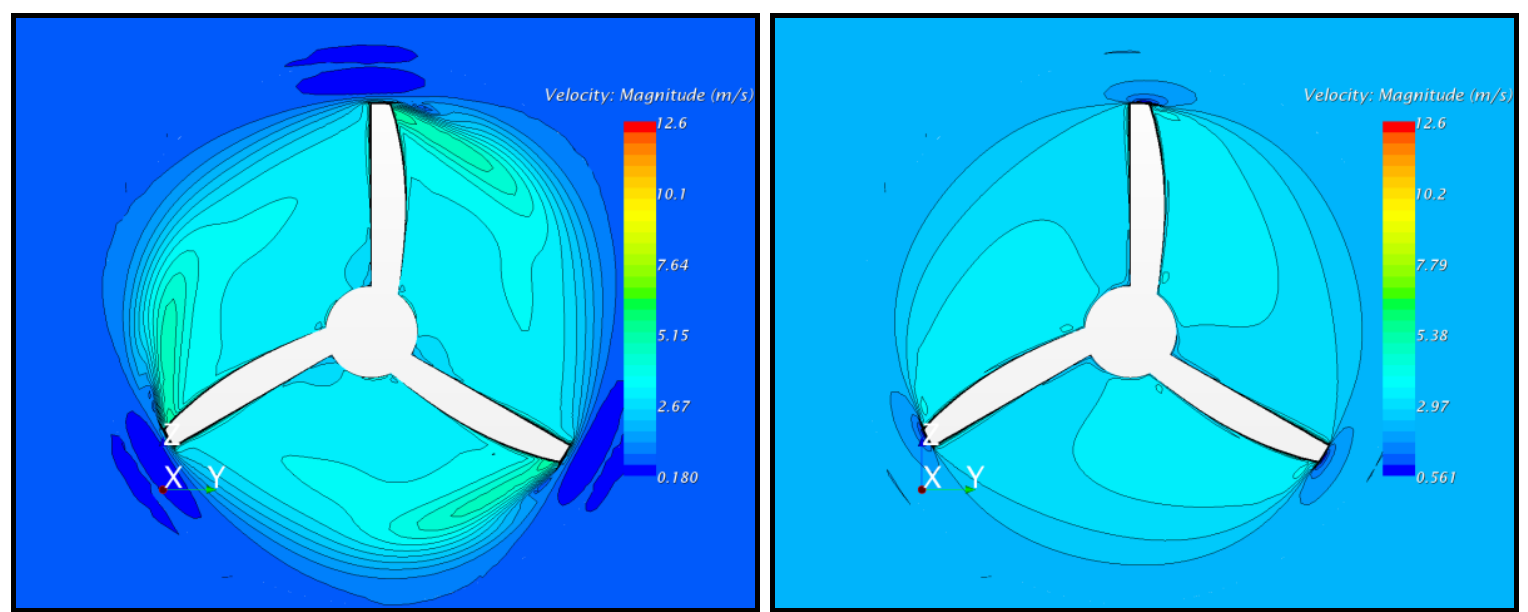

Fig. 16 Velocity distribution in the vertical plane at $J=0.2$ (left) and $J=0.55$ (right) 
The velocity distribution in the vertical plane of propeller with $P / D=0.8$ for two values of $J$ is shown in Figure 16. The larger difference in velocity distribution is present at lower $J$ value due to larger thrust at lower $J$ value.

The streamlines behind operating propeller with $P / D=0.8$ for two values of $J$ are shown in Figure 17. A larger trailing vortex can be noticed for lower $J$ value and therefore the open water efficiency is lower due to the loss of kinetic energy.
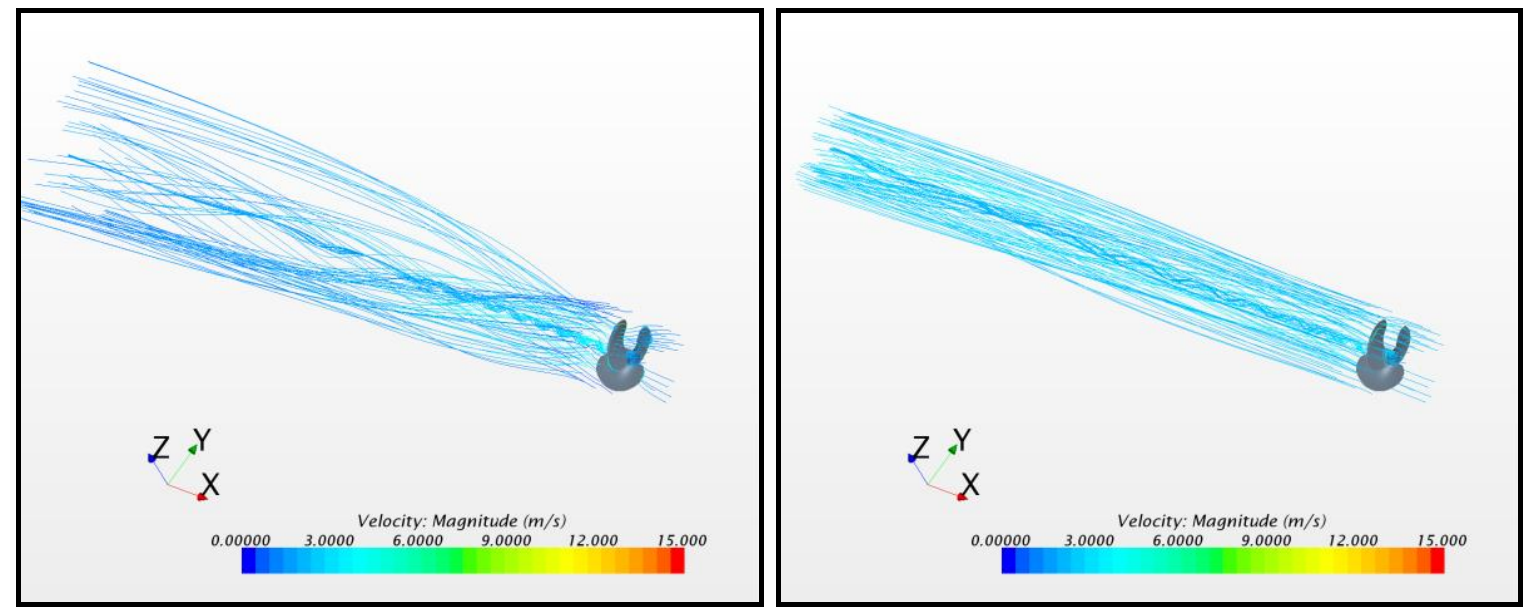

Fig. 17 Streamlines behind operating propeller at $J=0.2$ (left) and $J=0.55$ (right)

\section{Conclusion}

In this paper the numerical simulations based on potential and viscous flow theory were performed for five Gawn series propellers. The obtained numerical results of OWT were compared with the available regression polynomials. An input for numerical simulations based on the lifting line theory were the discrete values of the chord length of the blade sections at different radii. Within this research the applicability of lifting line theory in preliminary design of a screw propeller was investigated. Trends of hydrodynamic coefficients obtained using lifting line theory and regression polynomials are the same for the range of $J$ values where the propeller is moderately loaded, which is of great importance since the most of screw propellers operate in this range.

3D models for five Gawn series propellers were generated and used as an input data for RANS simulations. It was shown that trends of hydrodynamic coefficients obtained using RANS simulations and regression polynomials based on the experimental data are the same. Also, the detail analysis of the flow around screw propeller in OWT was performed. RANS simulations provide a complete insight into the flow around screw propeller, i.e. pressure and velocity distributions as well as streamlines. This is a valuable benefit of RANS simulations compared to simulations based on the lifting line theory.

The satisfactory agreement between numerically obtained results and the ones obtained using regression polynomials was achieved for both lifting line and viscous flow theory in the range of $J$ values where the propeller is moderately loaded. Therefore, OpenProp can be used as a practical and efficient tool in preliminary design of screw propellers as simulations based on the lifting line theory are significantly faster and simpler than RANS simulations. However, once the optimal geometry is obtained using lifting line theory, RANS simulations 
should be performed in order to obtain detail insight into the flow phenomena around the propeller.

\section{REFERENCES}

[1] Sambolek, M.: Propulzija broda, Brodarski institut, Zagreb, 2012.

[2] Bertram, V.: Practical ship hydrodynamics, Butterworth-Heinemann, Oxford, 2004.

[3] Epps, B.P. and Kimball, R.W.: OpenProp v3: Open-source software for the design and analysis of marine propellers and horizontal-axis turbines. 2013.

[4] Bal, S.: A practical technique for improvement of open water propeller performance, Proceedings of the Institution of Mechanical Engineers, Part M: Journal of Engineering for the Maritime Environment, Vol. 225, No. 4, 2011, pp. 375-386.

[5] Gaggero, S., Gonzalez-Adalid, J., Sobrino, M. P.: Design and analysis of a new generation of CLT propellers, Applied Ocean Research, Vol. 59, 2016, pp. 424-450. https://doi.org/10.1016/j.apor.2016.06.014

[6] Bertetta, D., Brizzolara, S., Gaggero, S., Viviani, M., Savio, L.: CPP propeller cavitation and noise optimization at different pitches with panel code and validation by cavitation tunnel measurements. Ocean engineering, 2012, Vol. 53, pp. 177-195. https://doi.org/10.1016/j.oceaneng.2012.06.026

[7] Lee K. J., Hoshino, T., Lee, J. H.: A lifting surface optimization method for the design of marine propeller blades, Ocean Engineering, Vol. 88, 2014, pp. 472-479. https://doi.org/10.1016/j.oceaneng.2014.07.010

[8] Paik, K. J.: Numerical study on the hydrodynamic characteristics of a propeller operating beneath a free surface, International Journal of Naval Architecture and Ocean Engineering, Vol. 9, No. 6, 2017, pp. 655-667. https://doi.org/10.1016/j.ijnaoe.2017.02.006

[9] Islam, M., Jahra, F., Doucet, M.: Optimization of RANS solver simulation setup for propeller open water performance prediction. In: ASME 2015 34th International Conference on Ocean, Offshore and Arctic Engineering. American Society of Mechanical Engineers, Newfoundland, Canada, 2015. https://doi.org/10.1115/OMAE2015-41954

[10] Califano, A. and Steen, S.: Numerical simulations of a fully submerged propeller subject to ventilation. Ocean engineering, Vol. 38, No. 14, 2011, pp. 1582-1599. https://doi.org/10.1016/j.oceaneng.2011.07.010

[11] Subhas, S., Saji, V.F., Ramakrishna, S., Das, H.N.: CFD analysis of a propeller flow and cavitation, International Journal of Computer Applications Vol. 55, No. 16, 2012, pp. 26-33.

[12] Prakash, S. and Nath, D. R.: A computational method for determination of open water performance of a marine propeller, International Journal of Computer Applications Vol. 58, No.12, 2012, pp. 1-5.

[13] Krasilnikov, V., Sun, J., Halse, K. H.: CFD investigation in scale effect on propellers with different magnitude of skew in turbulent flow, Proceedings of First International Symposium on Marine Propulsors (SMP'09), Trondheim, Norway, 2009.

[14] Müller, S.-B., Abdel-Maksoud, M., Hilbert, G.: Scale effects on propellers for large container vessels, Proceedings of First International Symposium on Marine Propulsors (SMP'09), Trondheim, Norway, 2009.

[15] Sungwook, L. and Paik, K.J.: URANS simulation of a partially submerged propeller operating under the bollard condition, Brodogradnja, Vol. 69, No.1, 2018, pp. 107-121. https://doi.org/10.21278/brod69107

[16] Paik, K. J.: Numerical study on the wake evolution of Contra-Rotating Propeller in propeller open water and self-propulsion conditions, Brodogradnja, Vol. 68, No. 1, 2017, pp. 125-138. https://doi.org/10.21278/brod68108

[17] Kinaci, O. K. and Gokce, M. K: A computational hydrodynamic analysis of Duisburg Test Case with free surface and propeller, Brodogradnja, Vol. 66, No. 4, 2015, pp. 23-38.

[18] Epps, B.P.: Openprop v2.4 Theory Document. Technical Report. MIT Department of Mechanical Engineering, 2010.

[19] Vlašić, D.: Numerički alat za preliminarni projekt brodskog vijka, Rad za Rektorovu nagradu, 2017.

[20] Ferziger, J.H. and Perić, M.: Computational Methods for Fluid Dynamics, Springer Science \& Business Media, Berlin, 2012.

[21] STAR-CCM+, User Guide, CD-adapco, 2016. 
[22] Farkas, A., Degiuli, N., Martić, I.: Assessment of hydrodynamic characteristics of a full-scale ship at different draughts, Ocean Engineering, Vol. 156, 2018, pp. 135-152. https://doi.org/10.1016/j.oceaneng.2018.03.002

[23] Carlton, J.: Marine Propellers and Propulsion, Second Edition, Elsevier Ltd., 2007.

Submitted: $\quad$ 09.05.2018. Deni Vlašić

Nastia Degiuli, nastia.degiuli@fsb.hr

Accepted: $\quad$ 27.07.2018. Andrea Farkas

Ivana Martić

University of Zagreb,

Faculty of Mechanical Engineering and Naval Architecture,

Ivana Lučića 5, 10000 Zagreb, Croatia 